\title{
Characterization of an Expressed Triticum monococcum Glu-Aly Gene Containing a Premature Termination Codon in its C-terminal Coding Region
}

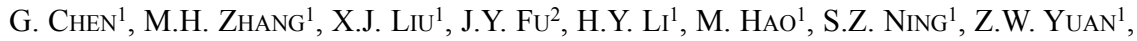 \\ Z.H. YAN ${ }^{1}$, B.H. WU ${ }^{1}$, D.C. LIU ${ }^{1}$ and L.Q. ZHANG ${ }^{1 *}$ \\ ${ }^{1}$ Triticeae Research Institute, Sichuan Agricultural University at Chengdu, Wenjiang, Sichuan, 611130, China \\ ${ }^{2}$ Institute of Ecological Agriculture, Sichuan Agricultural University at Chengdu, Wenjiang, \\ Sichuan 611130, China \\ (Received 1 April 2017; 24 September 2017; \\ Communicated by E. Kapusi)
}

\begin{abstract}
Premature termination codons (PTCs) are an important reason for the silence of highmolecular-weight glutenin subunits in Triticum species. Although the Glu-Aly gene is generally silent in common wheat, we here isolated an expressed Glu-Aly gene containing a PTC, named 1Ay8.3, from Triticum monococcum ssp. monococcum $\left(\mathrm{A}^{\mathrm{m}} \mathrm{A}^{\mathrm{m}}, 2 \mathrm{n}=2 \mathrm{x}=14\right)$. Despite the presence of a PTC (TAG) at base pair positions 1879-1881 in the C-terminal coding region, this did not obviously affect $1 A y 8.3$ expression in seeds. This was demonstrated by the fact that when the PTC TAG of $1 A y 8.3$ was mutated to the CAG codon, the mutant in Escherichia coli bacterial cells expressed the same subunit as in the seeds. However, in $E$. coli, 1Ay8.3 containing the PTC expressed a truncated protein with faster electrophoretic mobility than that in seeds, suggesting that PTC translation termination suppression probably occurs in vivo (seeds) but not in vitro (E. coli). This may represent one of only a few reports on the PTC termination suppression phenomenon in genes.
\end{abstract}

Keywords: high-molecular-weight glutenin subunits, premature termination codon, termination suppression

\section{Introduction}

High-molecular-weight glutenin subunits (HMW-GSs) play a key role in determining flour processing quality (Lawrence and Shepherd 1980; Shewry et al. 2002). In common wheat, HMW-GSs are encoded by locus Glu-A1 on chromosome arm 1AL, Glu-B1 on 1BL, and Glu-D1 on 1DL (Payne et al. 1987; Shewry et al. 1992). Each locus consists of two tightly linked genes encoding one $\mathrm{x}$-type subunit and one y-type subunit (Payne et al. 1987). The $\mathrm{x}$ - and $\mathrm{y}$-type subunits have similar primary structures, including a repetitive region and conserved $\mathrm{N}$-terminal and C-terminal domains (Shewry et al. 2002).

Although common wheat contains six different HMW-GS genes $(1 A x, 1 A y, 1 B x, 1 B y$, $1 D x$, and $1 D y$ ), only up to five subunits are typically observed since the $1 A y$ gene is always silent in common wheat (Forde et al. 1985; D’Ovidio et al. 1996). However, some

*Corresponding author; E-mail: zhanglianquan1977@126.com; Phone: 86-28-82650313; Fax: 86-28-82650350 
active 1 Ay genes have been identified in diploid and tetraploid wheats (Margiotta et al. 1996; Jiang et al. 2009; Guo et al. 2013; Li et al. 2015). The incorporation of an active 1 Ay subunit from wild wheat species into bread wheat could improve the gluten strength of wheat flour (Ciaffi et al. 1991; Alvarez et al. 2009).

Two mechanisms of $1 A y$ gene silencing have been reported in wheat. The first is transposon insertion leading to inactivation of the coding region. For example, the insertion of an 8.6-kb LTR retrotransposon into the coding region causes silencing of $1 A y$ gene expression in some tetraploid Triticum turgidum wheat and common wheat (Harberd et al. 1987). The second silencing mechanism is nonsense mutation leading to the generation of a premature termination codon (PTC) in the gene coding sequence (Forde et al. 1985; Jiang et al. 2009). In the present study, we found that a Glu-Aly gene from the diploid species Triticum monococcum was expressed even though it contains a PTC in its C-terminal coding region. We sought to characterize the expressed gene containing a PTC and examined its expression in vivo and in vitro.

\section{Materials and Methods}

\section{Plant materials}

The wheat $T$. monococcum ssp. monococcum accession $10-1\left(\mathrm{~A}^{\mathrm{m}} \mathrm{A}^{\mathrm{m}}, 2 \mathrm{n}=2 \mathrm{x}=14\right)$ was used in this study. HMW-GSs from the hexaploid common wheat Chinese Spring (CS) (null, 1Bx7 + 1By8, 1Dx2 + 1Dy12) were used as references for HMW-GS identification.

\section{SDS-PAGE analysis}

The HMW glutenin proteins were extracted and separated based on Yan et al. (2002). Seeds harvested over four successive years from 2012 to 2015 were analyzed to confirm the homozygosity on HMW-GS alleles in accession 10-1. At least five seeds for each year were analyzed.

\section{Cloning and sequencing of the HMW Glu-Aly gene ORF}

Genomic DNA was extracted from the young leaf tissues of T. monococcum accession 10-1 using a Plant Genomic DNA Kit (TIANGEN, Beijing, China). A pair of universal primers (PF: 5'-ATGGCTAAGCGGC/TTA/GGTCCTCTTTG-3' and PR: 5'-CTATCACTGGCTA/GGCCGACAATGCG-3') were used to amplify the complete coding region of the Glu-Aly gene following the method of Guo et al. (2013). PCRs were performed using a cycling program consisting of a denaturation cycle at $95{ }^{\circ} \mathrm{C}$ for $3 \mathrm{~min}$; 35 cycles of amplification $\left(95^{\circ} \mathrm{C}\right.$ for $30 \mathrm{sec}, 68^{\circ} \mathrm{C}$ for $30 \mathrm{sec}, 72{ }^{\circ} \mathrm{C}$ for $2 \mathrm{~min}$ per cycle), and a final extension cycle of $72{ }^{\circ} \mathrm{C}$ for $10 \mathrm{~min}$. The PCR products were separated on $1.5 \%$ agarose gels, and the expected fragments were recovered, purified, and ligated into a pMD19-T vector (TaKaRa, Dalian, China). The ligated mixtures were transformed into Escherichia coli Top10 competent cells. DNA sequencing was performed by TsingKe 
Biological Technology Company (Chengdu, China). Both the cloning and sequencing were repeated five times to exclude errors. Sequence alignment and analysis were carried out according to Li et al. (2015).

\section{Isolation of total RNA and 3'-RACE}

Total RNA was extracted from developing seeds at approximately 15 days post anthesis using a Plant Total RNA Kit V1.5 (BIOFIT, Chengdu, China) according to the manufacturer's instructions. An RNase-free DNase set (OMEGA, Shanghai, China) was then used to remove DNA. Purified RNA $(5 \mu \mathrm{g})$ was used to synthesize DNA using a SuperScript ${ }^{\mathrm{TM}}$ III First-Strand cDNA Synthesis Kit (Invitrogen, Carlsbad, CA, USA) following the user's manual, the cDNA samples were stored at $-80{ }^{\circ} \mathrm{C}$ as a template for cloning the complete cDNA of Glu-1Ay gene and their 3'end region. The PCR condition and procedure were the same as those used in genomic DNA described above.

In order to amplify the cDNA 3'end of the Glu-Aly gene, 3'-RACE was performed using a rapid amplification of cDNA Ends Kit (Invitrogen) according to the manufacturer's protocol. A gene-specific primer (3'-GSP: AGGCCAACAAACAGGACAAGTG), designed from the region upstream of the PTC in the Glu-Aly gene, was used for PCR amplification using Super-Fidelity DNA polymerase (Vazyme). The PCR program used was as follows: $95{ }^{\circ} \mathrm{C}$ for $3 \mathrm{~min}$, followed by 25 cycles of $94{ }^{\circ} \mathrm{C}$ for $15 \mathrm{~s}, 62{ }^{\circ} \mathrm{C} / 60{ }^{\circ} \mathrm{C}$ for $30 \mathrm{~s}$, and $72{ }^{\circ} \mathrm{C} 1 \mathrm{~min}$, and finally $72^{\circ} \mathrm{C}$ for $10 \mathrm{~min}$. To ensure sequencing accuracy, cDNA sequencing was repeated 30 times.

\section{Expression of the cloned Glu-Aly gene in E. coli}

In order to remove the signal peptide coding sequence from Glu-A1y ORFs, two pairs of primer combinations were used (F: 5'-AAACATATGGAAGGTGAGGCCTCTAGGC-3' with either R1: 5'-AAAGAATTCCTACGCCTTTGCCACCTTTGG-3' or R2: 5'-AAAGAATTCCTATCACTGGCTGGCCGAC-3'). F and R1 were used to amplify the sequence from the $\mathrm{N}$ terminus to the PTC TAG in the $\mathrm{C}$-terminal domain, which encodes 606 deduced amino acid residues (designated 1Ay606). F and R2 were used to amplify the full sequence, including the PTC, which encodes 629 deduced amino acid residues (designated 1Ay629). The restriction enzyme sites for NdeI and EcoRI (underlined) were introduced into the ORF for subsequent cloning. The resulting fragments were cloned into a pET-30a expression vector (Novagen). The recombinant constructs were transformed into E. coli strain BL21 (DE3) pLysS according to Li et al. (2015).

\section{Construction of the 1Ay8.3 mutant}

A 1Ay8.3 mutant, designated 1Ay629M, was constructed in pET-30a by replacement of the PTC TAG at base pair positions 1879-1881 with CAG. PCR was performed according to the site-directed mutagenesis protocol reported by Zheng et al. (2004). A pair of mutant primers (M-F: 5'-CCCAAAGGTGGCAAAGGCGCAGCATCCGACGGCA- 
CAG-3' and M-R: 5'- CTGTGCCGTCGGATGCTGCGCCTTTGCCACCTTTGGG-3') were used to obtain the 1 Ay8.3 mutant.

\section{Mass spectrometry analysis}

For matrix-assisted laser desorption ionization time of flight mass spectrometry (MALDI-TOF/MS) analysis, the target protein bands were manually excised from an SDSPAGE gel. And the excised products collected from eight gel bands for each sample. MALDI-TOF/MS assays were performed as suggested by Shu et al. (2011). The MALDITOF mass spectrometer analysis and parameters for database search were set by Sangon Biotechnology Company (Shanghai, China).

The protein bands excised from SDS-PAGE gel were also used in Nano Liquid Chromatography Mass Spectrometer (Nano LC-MS/MS) analysis, according to Sugiyama et al. (2007). Two digestion enzymes of Chymotrypsin and Glu-C (V8-E) were used in the analysis. The Nano LC-MS/MS analysis and parameters for database search were set by Ketian Science Company (Beijing, China).

\section{Plasmid construction and transient transformation in leaf protoplast}

To study the condition of expression of 1 Ay8.3 gene with a PTC in vivo, PA7-yellow fluorescent protein (YFP) vector was used to transfect transiently leaf protoplasts. Two constructs based on a YFP fusion were used. The first construct corresponded to the 1Ay649-YFP fusion, for which the complete cDNA of 1 Ay8.3 contains a premature termination codon (PTC) and signal peptide sequence without double stop codons was amplified by PCR with a pair of primer (TP-F: 5' -AAACTCGAGATGGCTAAGCGGC/TTA/ GGTCCTCTTTG-3'; TP-R1: 5'-AAAACTAGTCTGGCTRGCCGACAATGCG-3'). The second construct of the 1Ay626-YFP fusion contained the partial cDNA of $1 A y 8.3$ sequence from ATG to the PTC without the PTC by using primers (TP-F: 5' -AAACTCGAGATGGCTAAGCGGC/TTA/GGTCCTCTTTG-3'; TP-R2: 5'-AAAACTAGTCGCCTTTGCCACCTTTGGGCTG-3'). The restriction enzyme sites for XhoI and SpeI (underlined) were introduced into the ORF for subsequent cloning.

Protoplasts were isolated from leaves of 10-day-old seedlings from T. monococcum 10-1. Protoplast isolation and transient transformation followed the method of Yoo et al. (2007). The tranfected cells were maintained at $25^{\circ} \mathrm{C}$ in dark without shaking for 12 $16 \mathrm{~h}$ before observation by using an epifluorescence Olympus BX63 microscope.

\section{Results}

\section{SDS-PAGE analysis}

SDS-PAGE analysis indicated that the T. monococcum ssp. monococcum accession 10-1 expressed two HMW-GSs, an x-type subunit of slower mobility and a y-type subunit of faster mobility (Fig. 1a, lane 1). The electrophoretic mobility of the y-type subunit was 
between that of the $1 \mathrm{Bx} 7$ and $1 \mathrm{By} 8$ proteins of the common wheat cultivar Chinese Spring, and different from the previously identified 1 Ay $12 *, 1 \mathrm{Ay} 8 *$, and $1 \mathrm{Ay} 8.2$ in T. monococcum ssp. monococcum (Guo et al. 2013; Li et al. 2015). We designated the y-type subunit of accession 10-1 as 1Ay8.3.

\section{Sequence analysis of the 1 Ay8.3 gene}

The DNA fragment of gene $1 A y 8.3$ was amplified (Fig. 1b). The sequence of 1 Ay 8.3 was deposited in GenBank with the accession number KU207221. The same sequence was obtained using cDNA, indicating that $1 A y 8.3$ has no introns. A PTC of TAG was present at base pair positions $1879-1881$ in the C-terminal coding region (Fig. S1*). The sequence of $1 A y 8.3$ is highly similar to the previously sequenced Glu-A1y genes GQ184456 and HQ834309 from T. monococcum ssp. monococcum, with identities of 99\%. Moreover, the PTC TAG is present at the same positions in GQ184456 and HQ834309.
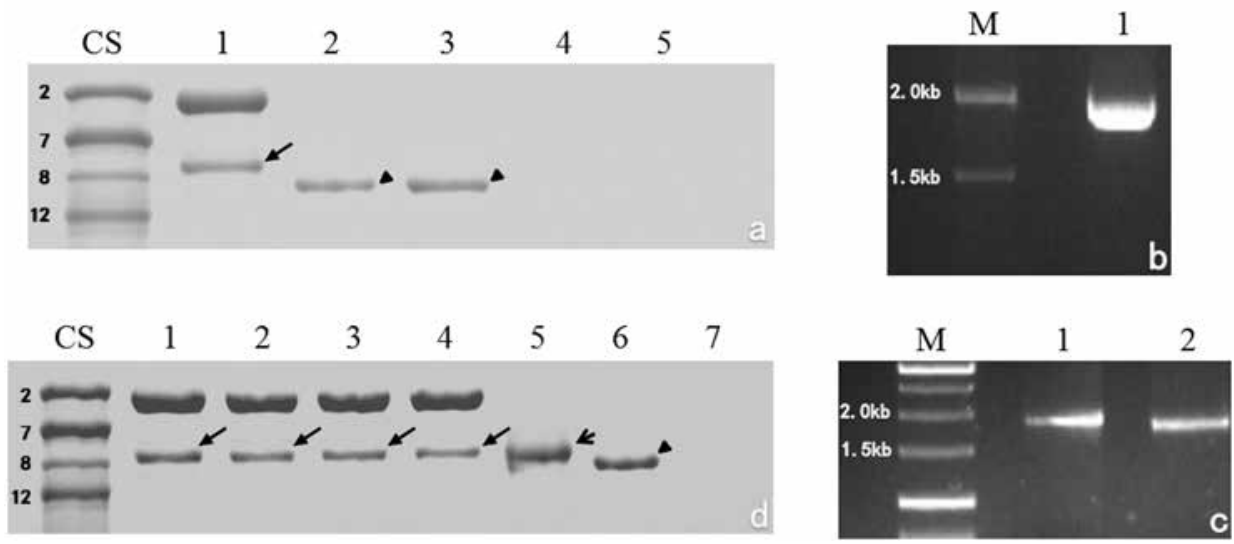

Figure 1. SDS-PAGE analysis and DNA amplification of 1Ay8.3 in Triticum monococcum ssp. monococcum accession 10-1. a SDS-PAGE profile of high-molecular-weight glutenin subunits from seeds (lane 1, the arrow indicates 1Ay8.3) and bacterial expression of the modified ORFs of 1Ay629 (lane 2) and 1Ay606 (lane 3) with isopropyl- $\beta$-D-thiogalactopyranoside (IPTG) induction, using 1Ay629 (lane 4) and 1Ay606 (lane 5) in bacterial cells without IPTG induction as controls. b PCR amplification of the complete ORFs of the 1Ay8.3 subunit (lane 1). c PCR amplifications of the ORFs from which the signal peptide sequence has been removed, including the premature termination codon (PTC) sequence (1Ay629) (lane 1) and terminating at the PTC sequence (1Ay606) (lane 2). d SDS-PAGE analysis of the 1Ay8.3 subunit (arrows) from seeds harvested in 2012 (lane 1), 2013 (lane 2), 2014 (lane 3), and 2015 (lane 4), and bacterial expression of 1Ay629M (lane 5) and 1Ay629 (lane 6) with IPTG induction, using 1Ay629M (lane 7) in bacterial cells without IPTG induction as a control.

CS, Chinese Spring; M, DNA markers

*Further details about the Electronic Supplementary Material (ESM) can be found at the end of the article. 


\section{Expression analysis of the 1Ay8.3 gene}

Although there was a PTC in the C-terminal domain, the 1 Ay8.3 gene was expressed in seeds (designated 1Ay629S). In order to analyze the effects of the PTC on the transcriptional level of $1 A y 8.3$, we sequenced its 3 '-UTR using the 3'-RACE technique (Fig. S2). All 30 transcripts analyzed contained the PTC at the same position as in the genomic DNA and corresponding cDNA sequences, confirming its normal transcription. We further investigated its expression in E. coli. Two fragments were amplified (Fig. 1c). Both of these can be expressed in E. coli. Although these fragments differed in length by $72 \mathrm{bp}$, their expressed products in $E$. coli showed no obvious differences. However, compared with the Glu-A1y protein from seeds (Fig. 1a, lane 1), these fragments had faster electrophoretic mobility (Fig. 1a, lane 2 and 3), indicating a difference between the in vivo (seeds) and in vitro (E. coli) expressions.

We further analyzed the HMW-GS locus of T. monococcum seeds harvested in four different years and deduced that the HMW-GS locus was homozygous, since all the analyzed protein samples extracted from seeds exhibited a consistent electrophoretic mobility (Fig. 1d, lanes 1-4). This observation excluded the possibility that the inconsistent expression between seeds and E. coli may have been caused by heterozygous alleles in the seeds used. We further mutated the $1 A y 8.3$ gene by substitution of the PTC TAG with $\mathrm{CAG}$ using a site-directed mutagenesis protocol. The $1 \mathrm{Ay} 8.3$ mutant, $1 \mathrm{Ay} 629 \mathrm{M}$, was successfully expressed in E. coli (Fig. 1d, lane 5). Although its expression product showed no obvious size difference compared with that of the seed protein, it did have slower electrophoretic mobility than the expressed wild-type sequence containing the PTC TAG (Fig. 1d, lane 6).

On the basis of MALDI-TOF/MS and Nano LC-MS/MS analysis, the expression products from seeds and E. coli, including the wild-type TAG PTC (1Ay629) and its mutant (1Ay629M), were confirmed to be 1Ay. Since some peptide sequences were detected (Fig. S3, Table S1). MALDI-TOF/MS analysis also showed that the glutenin proteins expressed in seeds and from the artificial 1 Ay8.3 mutant in E. coli exhibited a similar size of approximately $70 \mathrm{kDa}$, which is higher than that of 1 Ay8.3 expressed in E. coli (approx. $68 \mathrm{kDa}$ ) (Table $\mathrm{S} 1$ ). This also indicated that the 1 Ay8.3 expressed in E. coli was a truncated Glu-A1y protein.

\section{Transient expression of 1 Ay8.3 in leaf protoplast cells}

The 1Ay649-YFP (Fig. S4a) and 1Ay626-YFP (Fig. S4b) nucleotide sequences were introduced into the PA7-YFP plasmid under the control of the cauliflower mosaic virus 35S promoter and terminator. After obtaining high viability leaf protoplasts from $T$. monococcum ssp. monococcum 10-1, we investigated their possibility to express the 1Ay649-YFP and 1Ay626-YFP protein in fusion with YFP. After transient transformation of leaf protoplasts and $12 \mathrm{~h}$ of incubation in the dark, expression of the green fluorescent proteins was observed by epifluorescence microscope. The YFP alone was expressed in some leaf protoplasts and used as positive control (Fig. S5a). We observed the green fluorescence 
signals both for 1Ay649-YFP (Fig. S5b) and 1Ay626-YFP (Fig. S5c). The expression of 1Ay649-YFP having the PTC suggested the existence of PTC translation termination suppression also in leaf protoplast cells.

\section{Discussion}

Nonsense mutation generating a premature termination codon (PTC) in a coding sequence is a common phenomenon in gene silencing (Rehwinkel et al. 2006). Previous studies have suggested that PTCs seem to be an important factor involved in the silencing of HMW-GS genes, including the Glu-Aly loci (D'Ovidio et al. 1996; Guo et al. 2013; Li et al. 2015). In the current study, however, we identified a Glu-Aly gene $1 A y 8.3$ that was expressed, despite the fact that it contained a PTC at base pair positions 1879-1881 (Fig. S1). On the basis of the protein product size, it appears that seeds expressed an intact 1Ay8.3 subunit, whereas E. coli expressed a truncated protein (Figs. 1a and 1d). This suggested that termination suppression during translation probably occurred in vivo (seeds) but not in vitro (E. coli). Such PTC termination suppression has been observed in several organisms (Bertram et al. 2001).

Comparison with 15 previously sequenced $1 A y$ genes from common wheat and other Triticum species that contain a PTC in their coding region, revealed that a PTC similar to that identified in $1 A y 8.3$ is also contained in three other T. monococcum genes (Table S2), indicating a common origin. The PTCs in the remaining 12 genes are present in the $\mathrm{N}$-terminal domain or central repetitive region. Although genes from common wheat (T. aestivum) containing PTCs have been confirmed to be silent (Forde et al. 1985), it is unclear whether similar genes are expressed in other species. To our best knowledge, 1 Ay8.3 is the first HMW-GS gene in which potential PTC termination suppression of translation has been reported. However, at present, the underlying mechanisms remain unclear. Several natural mechanisms of termination suppression have been reported, including suppressor tRNAs (aminoacylated tRNAs with anticodons complementary to stop codons in mRNA), ribosomal frameshifting, and stop codon readthrough (see review by Dabrowski et al. 2015). In addition, nonsense-mediated mRNA decay (NMD) is a eukaryotic surveillance mechanism that eliminates mRNA containing a PTC to prevent the accumulation of truncated and potentially destructive proteins (Mendell et al. 2004). In this regard, a previous study has shown that a long 3'UTR was preferentially targeted to potentiate degradation by the NMD effector Upf1 (up-frameshift protein) in transcripts (Hogg and Goff 2010). The position of C-terminal coding region is closer to 3'UTR region than that of central repeat region. Further work is necessary in order to determine whether the position of the PTC is related to termination suppression of the $1 A y 8.3$ gene.

In addition, the expressed 1 Ay 8.3 contains a cysteine residue, which is present between the PTC TAG and two stop codons in the C-terminal domain. (Fig. S2), and thus the resulting protein from seeds contains seven cysteine residues. The extra cysteine residue may be useful for improving flour processing quality by enhancing dough strength. Earlier studies have reported an extra cysteine residue in the central repetitive region of the 
subunits $1 \mathrm{Dx} 5$ and $1 \mathrm{Ax} 2 * \mathrm{~B}$, which facilitate the formation of additional disulfide bonds, and thereby positively affects dough strength (Lafiandra et al. 1993; Juhász et al. 2003).

In our work, an expressed Glu-Aly allele 1Ay8.3 containing a premature termination codon (PTC) in its C-terminal coding region was identified from Triticum monococcum ssp. monососсит. Furthermore, it was suggested that termination suppression during translation probably occurs in vivo (seeds) but not in vitro (E. coli). This PTC termination suppression event of 1Ay8.3 could be used to investigate the pseudogenes of Glu-A1y and other HMW-GS caused by a PTC in its coding region in future, and further enrich the study of the mechanism of "Expression-Silence" for Glu-Aly gene in common wheat and other Triticum species.

\section{Acknowledgements}

This research was supported by the National Natural Science Foundation of China (31671682, 31671689) and Major International (Regional) Joint Research Project (31661143007).

\section{References}

Alvarez, J.B., Caballero, L., Nadal, S., Ramírez, M.C., Martín, A. 2009. Development and gluten strength evaluation of introgression lines of Triticum urartu in durum wheat. Cereal Res. Commun. 37:243-248.

Bertram, G., Innes, S., Minella, O., Richardson, J., Stansfield, I. 2001. Endless possibilities: translation termination and stop codon recognition. Microbiol. 147:255-269.

Ciaffi, M., Benedettelli, S., Giorgi, B., Porceddu, E., Lafiandra, D. 1991. Seed storage proteins of Triticum turgidum ssp. dicoccoides and their effect on the technological quality in durum wheat. Plant Breed. 107:309-319.

Dabrowski, M., Bukowy-Bieryllo, Z., Zietkiewicz, E. 2015. Translational readthrough potential of natural termination codons in eucaryotes-the impact of RNA sequence. RNA Biol. 12:950-958.

D’Ovidio, R., Masci, S., Porceddu, E. 1996. Sequence analysis of the 5'non-coding regions of active and inactive $1 A y$ HMW glutenin genes from wild and cultivated wheats. Plant Sci. 114:61-69.

Forde, J., Malpica, J.M., Halford, N.G. et al. 1985. The nucleotide sequence of a HMW glutenin subunit gene located on chromosome 1A of wheat (Triticum aestivum L.). Nucleic. Acids. Res. 13:6817-6832.

Guo, X.H., Wu, B.H., Hu, X.G. et al. 2013. Molecular characterization of two y-type high molecular weight glutenin subunit alleles $1 \mathrm{Ay} 12^{*}$ and $1 \mathrm{Ay} 8^{*}$ from cultivated einkorn wheat (Triticum monococcum ssp. monococcum). Gene 516:1-7.

Harberd, N.P., Flavell, R.B., Thompson, R.D. 1987. Identification of a transposon-like insertion in a Glu-1 allele of wheat. Mol. Gen. Genet. 209:326-332.

Hogg, J.R., Goff, S.P. 2010. Upf1 senses 3'UTR length to potentiate mRNA decay. Cell 143:379-389.

Jiang, Q.T., Wei, Y.M., Wang, F. et al. 2009. Characterization and comparative analysis of HMW glutenin $1 \mathrm{Ay}$ alleles with differential expressions. BMC Plant Biol. 9:16.

Juhász, A., Larroque, O.R., Tamás, L. et al. 2003. Bánkúti 1201 - an old Hungarian wheat variety with special storage protein composition. Theor. Appl. Genet. 107:697-704.

Lafiandra, D., D’Ovidio, R., Porceddu, E., Margiotta, B., Colaprico, G. 1993. New data supporting high Mr glutenin subunit 5 as determinant of quality differences among the pairs $5+10$ vs. $2+12$. J. Cereal Sci. 18:197-205.

Lawrence, G.J., Shepherd, K.W. 1980. Variation in glutenin protein subunits of wheat. Aust. J. Biol. Sci. 33:221-233. 
Li, Z.L., Li, H.Y., Chen, G. et al. 2015. Characterization of a novel y-type HMW-GS with eight cysteine residues from Triticum monococcum ssp. monococcum. Gene. 573:110-114.

Margiotta, B., Urbano, M., Colaprico, G., et al. 1996. Detection of y-type subunit at the Glu-A1 locus in some Swedish bread wheat lines, J. Cereal Sci. 23:203-211.

Mendell, J.T., Sharifi, N.A., Meyers, J.L., Martinez-Murillo, F., Dietz, H.C. 2004. Nonsense surveillance regulates expression of diverse classes of mammalian transcripts and mutes genomic noise. Nat. Genet. 36:1073-1078.

Payne, P.I., Nightingale, M.A., Krattiger, A.F,, Holt, L.M. 1987. The relationship between HMW glutenin subunit composition and the bread-making quality of British-grown wheat varieties. J. Sci. Food Agric. 40:51-65.

Rehwinkel, J., Raes, J., Izaurralde, E. 2006. Nonsense-mediated mRNA decay: target genes and functional diversification of effectors. Trends Biochem. Sci. 31:639-646.

Shewry, P.R., Halford, N.G., Belton, P.S., Tatham, A.S. 2002. The structure and properties of gluten: an elastic protein from wheat grain. Philos. Trans. Biol. Sci. 357:133-142.

Shewry, P.R., Halford, N.G., Tatham, A.S. 1992. High molecular weight subunits of wheat glutenin. J. Cereal Sci. 15:105-120.

Shu, L.B., Lou, Q.J., Ma, C.F. et al. 2011. Genetic, proteomic and metabolic analysis of the regulation of energy storage in rice seedlings in response to drought. J. Proteomics 11:4122-4138.

Sugiyama, N., Masuda, T., Shinoda, K. et al. 2007. Phosphopeptide enrichment by aliphatic hydroxy acidmodified metal oxide chromatography for nano-LC-MS/MS in proteomics applications. Mol. Cell. Proteomics 6:1103-1109.

Yan, Z.H., Wan, Y.F., Liu, K.F. et al. 2002. Identification of a novel HMW glutenin subunit and comparison of its amino acid sequence with those of homologous subunits. Chinese Sci. Bull. 47:220-225.

Yoo, S.D., Cho, Y.H., Sheen, J. 2007. Arabidopsis mesophyll protoplasts: a versatile cell system for transient gene expression analysis. Nat. Protoc. 2:1565-1572.

Zheng, L., Baumann, U., Reymond, J.L. 2004. An efficient one-step site-directed and site-saturation mutagenesis protocol. Nucleic Acids Res. 32:e115.

\section{Electronic Supplementary Material (ESM)}

Electronic Supplementary Material (ESM) associated with this article can be found at the website of CRC at http://www.akademiai.com/content/120427/

Electronic Supplementary Table S1. MALDI-TOF/MS and Nano LC-MS/MS identification of protein bands from SDS-PAGE gels

Electronic Supplementary Table S2. Positions of the PTCs in the coding region of 15 Glu-A1y genes

Electronic Supplementary Figure S1. Position of the premature termination codon (PTC) in 1Ay8.3

Electronic Supplementary Figure S2. Sequence alignments of the $1 A y 8.3$ gene amplified from genomic DNA and cDNA. *: stop codon; Cys: Cysteine

Electronic Supplementary Figure S3. The detected peptide sequences from 1 Ay8.3 by mass spectrometry. The marked peptide sequences were identified by MALDI-TOF/MS using the digestion enzyme trypsin (red) and by Nano LC-MS/MS using enzyme Glu-C (green) and chymotrypsin (purple and underlined)

Electronic Supplementary Figure S4. Gene structures for fusion protein. a 1Ay649-YFP, the full cDNA sequence of $1 A y 8.3$ including the signal peptide sequence and the premature termination codon (PTC) TAG but without the double stop codons. b 1Ay626-YFP, the partial cDNA of 1Ay8.3 sequence from signal peptide sequence to the PTC but without the PTC 
Electronic Supplementary Figure S5. Transient transformation of the 1Ay8.3 fusion protein in leaf protoplast cells of diploid wheat 10-1. a Leaf protoplasts were transiently transformed with a plasmid encoding the YFP as a positive control. $b$ The sequence of 1Ay649-YFP was transiently expressed in some leaf cells. c The sequence of 1Ay626-YFP was transiently expressed in some leaf cells. The red fluorescence was used to detect the spontaneous signal from chloroplast of leaf. Merged images of YFP as well as bright filed images of protoplasts were shown. Scale bar $100 \mu \mathrm{m}$. Experiment repetition $\mathrm{n}=3$ 